\title{
Velocity-independent estimation of kinematic attributes in vertical transverse isotropy media using local slopes and predictive painting
}

\author{
M. Javad Khoshnavaz ${ }^{1}$, Andrej Bóna ${ }^{1}$, and Milovan Urosevic ${ }^{1}$
}

\begin{abstract}
Agood seismic velocity model is required for many routine seismic imaging techniques. Velocity model building from seismic data is often labor intensive and time consuming. The process becomes more complicated by taking nonhyperbolic traveltime estimations into account. An alternative to the conventional time-domain imaging algorithms is to use techniques based on the local event slopes, which contain sufficient information about the traveltime moveout for velocity estimation and characterization of the subsurface geologic structures. Given the local slopes, there is no need for a prior knowledge of a velocity model. That is why the term "velocity independent" is commonly used for such techniques. We improved upon and simplified the previous versions of velocity-independent nonhyperbolic approximations for horizontally layered vertical transverse isotropy (VTI) media by removing one order of
\end{abstract}

differentiation with respect to offset from the imaging kinematic attributes. These kinematic attributes are derived in terms of the local event slopes and zero-offset two-way traveltime (TWTT). We proposed the use of predictive painting, which keeps all the attributes curvature independent, to estimate the zero-offset TWTT. The theoretical contents and performance of the proposed approach were evaluated on synthetic and field data examples. We also studied the accuracy of moveout attributes for shifted hyperbola, rational, three-parameter, and acceleration approximations on a synthetic example. Our results show that regardless of the approximation types, NMO velocity estimate has higher accuracy than the nonhyperbolicity attribute. Computational time and accuracy of the inversion of kinematic attributes in VTI media using our approach were compared with routine/conventional multiparameter semblance inversion and with the previous velocityindependent inversion techniques.

\section{INTRODUCTION}

In many conventional seismic imaging techniques, a seismic velocity model is a requirement. The model is often built through velocity analysis from seismic data. This is still one of the most critical stages in seismic data processing (Yilmaz, 2001), and the velocity errors influence the accuracy of the final image. Routine velocity analysis methods from seismic data (i.e., constant velocity stack and semblance analysis) are generally labor-intensive and time-consuming. An experienced processor is generally required to pick the proper velocities in the velocity spectrum. Velocity analysis is often more time-consuming by taking the anisotropy and/or lateral inhomogeneity into account, when more than a single parameter is required for nonhyperbolic moveout approximations (Alkhalifah and Tsvankin, 1995).

Oriented/velocity-independent time-domain imaging using local slopes is an alternative to the routine imaging workflows. Local slopes represent the apparent ray parameters or apparent slownesses in the seismic record. They carry information about the local traveltime moveout that is defined by the subsurface geologic structures (Fomel, 2007a). Over the past 80 years, many advances have been made in velocity-independent imaging. The idea of using local slopes from prestack data goes back to the works done by Rieber (1936) and Riabinkin (1957). Ottolini (1983) derived the migration velocity for each point in the registered seismic data to apply it on his velocity-independent migration algorithm for horizontal interfa-

\footnotetext{
Manuscript received by the Editor 17 November 2015; revised manuscript received 30 March 2016; published online 11 August 2016.

${ }^{1}$ Curtin University, Department of Exploration Geophysics, Perth, Western Australia, Australia and Deep Exploration Technologies Cooperative Research Centre, Australia. E-mail: mj.khoshnavaz@postgrad.curtin.edu.au; a.bona@curtin.edu.au; m.urosevic@curtin.edu.au.

(C) 2016 Society of Exploration Geophysicists. All rights reserved.
} 
ces. Fomel (2007a) generalized this idea by developing analytical velocity-independent techniques to transform seismic data from prestack domain to the image domain for different time-domain imaging operators. Cooke et al. (2009) further developed a local slope-based prestack time migration technique and used the oriented migration velocities to remove multiples. Bóna (2011) developed another oriented/velocity-independent prestack time migration technique for linear reflectors in common-source domain. He used the local slopes and the second derivatives of two-way traveltime (TWTT) with respect to offset (curvatures). All these techniques are developed based on the time-domain imaging premise of the hyperbolic traveltime moveout.

Direct use of local slopes in the estimation of interval velocities for anisotropic media with polar anisotropy (vertical transverse isotropy [VTI]) was proposed by Fowler et al. (2008). Velocity-independent time imaging was extended to anisotropic medium by Burnett and Fomel (2009a, 2009b). Their technique is designed for 3D elliptically anisotropic media. Casasanta and Fomel (2011) developed another velocity-independent time imaging technique for VTI media in the slant-stack $(\tau-p)$ domain. Stovas and Fomel (2016) proposed the use of local slopes to obtain kinematic attributes, including normal moveout (NMO) velocity, anisotropy anellipticity coefficient $\eta$, and zero-offset TWTT $t_{0}$ for different nonhyperbolic approximations. These include shifted hyperbola approximation (Malovichko, 1978; de Bazelaire, 1988; Castle, 1994), rational nonhyperbolic approximation (Alkhalifah and Tsvankin, 1995), and generalized moveout approximation (Fomel and Stovas, 2010). To find the parameters describing the different moveout approximations, Stovas and Fomel (2016) proposed to estimate the curvature of the wavefront, or equivalently the second derivative of the traveltime with respect to offset; however, the curvature estimation is very difficult to achieve with the required accuracy. These difficulties are described in the "Discussion" section of this paper.

To avoid the curvature estimation, we further developed the research of Stovas and Fomel (2016) and derived the kinematic attributes, nonhyperbolicity parameter, and NMO velocity, in terms of

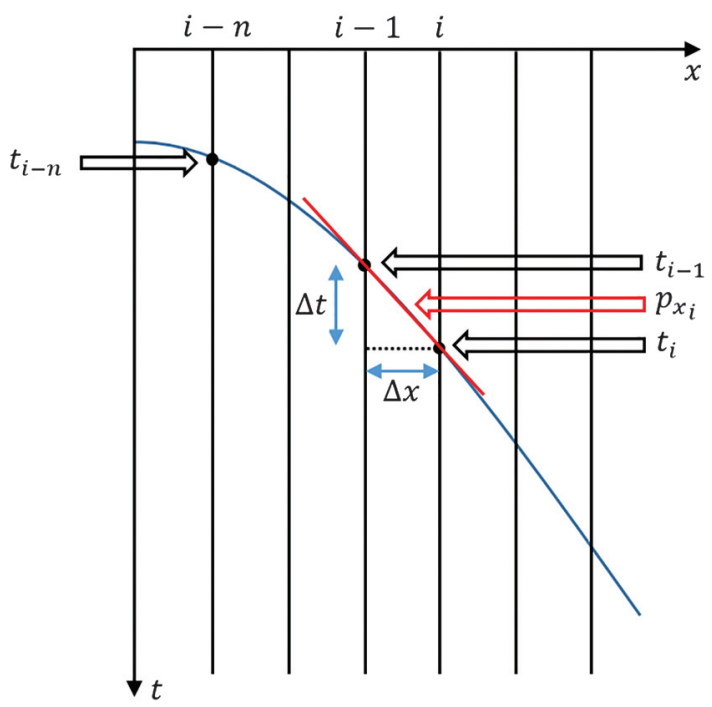

Figure 1. Predictive painting of a seismic event using local slopes. Black dots show the samples belonging to the same seismic wavefront. The red line indicates the local slope between the two neighboring samples. local slopes and zero-offset TWTTs for different nonhyperbolic approximations, including shifted-hyperbola (Malovichko, 1978; de Bazelaire, 1988; Castle, 1994), rational (Alkhalifah and Tsvankin, 1995), three-parameter (Blias, 2009), and acceleration approximations (Taner et al., 2005). For rational approximation, we used the expressions derived by Stovas and Fomel (2016). In our approach, the need for the curvature estimation is replaced by the estimation of zero-offset TWTT using the predictive painting approach (Fomel, 2010); therefore, all kinematic attributes stay curvature independent.

Because the generalized moveout approximation requires more parameters than the other approximations, Stovas and Fomel (2016) proposed to use a two-point-to-point mapping technique, where the slopes and curvatures are computed at two points on the same wavefront; however, having the zero-offset TWTT of the wavefront, it is possible to use a one-point-to-point scanning similar to the approach we proposed for other approximations in this paper.

To show the applicability of the proposed approach to the various approximations, we compared the accuracy of the estimated moveout attributes on a synthetic multilayered VTI CMP gather. Regardless of the approximation type, NMO velocity has the highest accuracy, whereas nonhyperbolicity attribute $\eta$ is less accurate. Similar to the observations of Asgharzadeh et al. (2014), we found that among the tested approximations, the rational approximation has the highest accuracy, whereas the approximation of Taner et al. (2005) has the lowest. Computational time and accuracy in the inversion of kinematic attributes in VTI media using the proposed approach are compared with routine/conventional multiparameter semblance inversion and with the previous velocity-independent inversion techniques. To estimate the local slopes and zero-offset TWTTs, we used the plane-wave destructor and predictive painting (Fomel, 2002, 2010) available in Madagascar open-source multidimensional data analysis package (Fomel et al., 2013).

\section{PREDICTIVE PAINTING}

The main concept of predictive painting, introduced by Fomel (2010), is to predict each seismic event from its neighbors that is shifted along the local event slopes. Local slopes are often estimated by measuring the time difference or time shift between two points carried by a seismic wavefront. Given the local slopes, one can implement an inverse process to find the time shift between two neighboring traces. The prediction process can be explained in the following way.

Figure 1 shows a typical seismic event that can be either hyperbolic or nonhyperbolic. The event can be located in any seismic domain (e.g., CMP and common-source domain). Consider a sample located at trace number $i$ with the TWTT of $t_{i}$. Finding the TWTT of the seismic event at trace number $i-n$ is desired. The first step is to predict the TWTT of the event at the neighboring trace number $i-1$. It is done by defining a predictive operator, which is in terms of the local slope $p$ between the two samples. The $\Delta t$ and $\Delta x$ denote the time shift and receiver spacing, respectively. The slope is shown by the red line in Figure 1 .

The tangent of the angle between the slope and the horizontal dashed line determines the predictive operator as follows:

$$
\tan \gamma=p_{x i}=\frac{\Delta t}{\Delta x}=\frac{t_{i}-t_{i-1}}{\Delta x},
$$




$$
t_{i-1}=t_{i}-p_{x i} \Delta x
$$

Writing and repeating the above operator until the desired trace $(i-n)$, summation and simplification of the results gives the general predictive operator that is given by

$$
t_{i-n}=t_{i}-\left(\sum_{k=0}^{n-1} p_{x i-k}\right) \Delta x
$$

For zero-offset TWTT $\left(t_{0}\right), n$ will be the trace number difference between the zero-offset trace and the $i$ th trace. This velocity-independent moveout equation remains the same for all hyperbolic and nonhyperbolic events.

\section{KINEMATIC ATTRIBUTE ESTIMATIONS}

\section{Shifted hyperbola approximation}

Shifted hyperbola approximation (Malovichko, 1978; de Bazelaire, 1988; Castle, 1994) is one of the most popular moveout approximations for explaining nonhyperbolic moveouts, and it is given by

$$
t \cong t_{0}\left(1-\frac{1}{s}\right)+\frac{1}{s} \sqrt{t_{0}^{2}+\frac{s x^{2}}{v^{2}}}
$$

where $t, t_{0}$, and $v$ denote TWTT, zero-offset TWTT, and the NMO velocity, respectively. Parameter $s$ is related to anisotropy and/or heterogeneity of seismic structures and velocities. Differentiation of equation 4 with respect to offset $x$ gives the local slope $p$

$$
p=\frac{x}{v^{2} \sqrt{t_{0}^{2}+\frac{s x^{2}}{v^{2}}}} .
$$

Equations 4 and 5 construct a system of equations with two unknown variables, $s$ and $v$. Solution of the system gives $s$ and $v$ in terms of zero-offset TWTT and the local slopes

$$
\begin{gathered}
s=-\frac{t_{0}\left(x p-2 t+2 t_{0}\right)}{\left(t-t_{0}\right)\left(x p-t+t_{0}\right)}, \\
v^{2}=\frac{x\left(x p-t+t_{0}\right)}{t_{0} p\left(t-t_{0}\right)} .
\end{gathered}
$$

Golikov and Stovas (2012) rearranged different moveout approximations in terms of $\eta$ for VTI media. For shifted hyperbola approximation, $\eta$ is expressed by

$$
\eta=\frac{s-1}{8} .
$$

Note that the values of $\eta$ represent nonhyperbolicity of the moveout.

\section{Rational approximation}

Rational moveout approximation (Alkhalifah and Tsvankin, 1995) has the form of

$$
t^{2} \cong t_{0}^{2}+\frac{x^{2}}{v^{2}}-\frac{2 \eta x^{4}}{v^{4} t_{0}^{2}\left[1+(1+2 \eta) \frac{x^{2}}{v^{2} t_{0}^{2}}\right]} .
$$

Kinematic attributes derived by Stovas and Fomel (2016) are given by

$$
\begin{gathered}
\eta=\frac{t_{0}^{2}\left(1+R^{2}\right)\left(t^{2}-t_{0}^{2}-p x t\right)}{2 R^{2}\left(t^{2}-t_{0}^{2}\right)^{2}}, \\
v^{2}=\frac{R x^{2}}{t_{0}^{2}},
\end{gathered}
$$

where

$$
R=\frac{p x t t_{0}^{2}+t_{0} \sqrt{\left(p x t t_{0}\right)^{2}-4\left(t^{2}-t_{0}^{2}\right)^{2}\left(t^{2}-t_{0}^{2}-p x t\right)}}{2\left(t^{2}-t_{0}^{2}\right)^{2}} .
$$

In their technique, zero-offset TWTT that is in terms of the wavefront curvature or the second derivative of TWTT with respect to offset, must first be estimated to estimate the other kinematic attributes. It is done by solving a complicated fourth-degree polynomial for $t_{0}^{2}$. In our approach, this need is replaced by the estimation of the zero-offset TWTT using predictive painting.

\section{Three-parameter approximation}

Blias $(2007,2009)$ introduced several three-parameter nonhyperbolic moveout approximations for inhomogeneous media. We formulated all of them in terms of local slopes and zero-offset TWTT. For brevity, only his second approximation is included in this work. The moveout approximation is expressed by

$$
t^{2} \cong \frac{t_{0}^{2}}{2}+\frac{x^{2}}{v^{2}}+\frac{1}{2} \sqrt{t_{0}^{4}+\frac{2 A x^{4}}{v^{4}}}
$$

where $A$ defines the inhomogeneity. The kinematic attributes are derived by a similar analysis to shifted hyperbola approximation. The attributes are given by the following equations:

$$
\begin{gathered}
A=\frac{2(2 t p x c-1)\left(2 b c-x^{2}\right)}{x^{2}}, \\
v^{2}=2 x^{2} c,
\end{gathered}
$$

where

$$
\begin{gathered}
b=t^{2}-\frac{t_{0}^{2}}{2}, \\
c=\frac{2\left(p t x-t^{2}\right)+t_{0}^{2}}{4\left(p t b x-t^{4}+t^{2} t_{0}^{2}\right)} .
\end{gathered}
$$

Based on the reformulation of the moveout approximation by Golikov and Stovas (2012), $\eta$ is given by 


$$
\eta=-\frac{A}{4}
$$

\section{Acceleration approximation}

Increase of velocity with offset was interpreted as acceleration by Taner et al. (2005, 2007). They provided two nonhyperbolic moveout approximations by adding an extra term (acceleration) to the classic NMO equation. The accuracy of the proposed approximations is comparable with three-parameter nonhyperbolic approximations (Golikov and Stovas, 2012). The second moveout approximation from Taner et al. (2005) is expressed by

$$
t^{2} \cong t_{0}^{2}+\frac{x^{2}}{v^{2}\left(1+\frac{2 \eta x^{2}}{v^{2} t_{0}^{2}}\right)}
$$

Following a similar analysis to the previous part, the kinematic attributes are derived and expressed by

$$
\begin{gathered}
\eta=-\frac{1}{2} \frac{t_{0}^{2}\left(p t x+t_{0}^{2}-t^{2}\right)}{\left(t_{0}^{2}-t^{2}\right)^{2}}, \\
v^{2}=\frac{p t x^{3}}{\left(t_{0}^{2}-t^{2}\right)^{2}} .
\end{gathered}
$$

\section{APPLICATION TO A SYNTHETIC DATA EXAMPLE}

In this example, the proposed inversion technique is applied to a synthetic CMP gather from a horizontally layered model including 12 VTI layers. The NMO velocities increase linearly with depth/ time with the range of 1800-2300 m/s, and the effective nonhyperbolicity parameter $\eta$ varies between 0.04 and 0.16 (Table 1 ).

Figure 2a-2c shows the isotropic CMP gather, anisotropic CMP gather, and the amplitude difference between them, respectively. The traveltimes used to generate the gather were computed from effective VTI ray velocities corresponding to the given $\eta$ s following similar approach described in Bóna et al. (2008). Figure 2d and 2e shows the local slopes and zero-offset TWTTs estimated by planewave destructor (Fomel, 2002) and predictive painting (Fomel, 2010) in Madagascar Open-Source multidimensional data analysis package (Fomel et al., 2013), respectively. Figure $2 \mathrm{f}$ shows the result of flattening of the anisotropic gather shown in Figure $2 b$ by the application of the predictive painting technique. It is observed that the anisotropic data are well-flattened.

To quantify the accuracy of the predicted TWTTs, including the zero-offset TWTTs, the exact TWTT curve at interface 5 obtained by forward modeling is compared with its corresponding predicted TWTT in Figure 3. Predicted TWTT curve is obtained using the fact that TWTTs with the same zero-offset TWTT belong to the same wavefront. The procedure of predicting TWTT curves from the predicted zero-offset TWTTs is known as time warping (Burnett and Fomel, 2009b). The standard error around the exact curve is less than $0.1 \%$ in this example.

In the next step, the estimated local slopes and zero-offset TWTTs are used to measure the nonhyperbolicity (anisotropy anellipticity) coefficient $\eta$ and NMO velocity from the above approx- imations. Figure $4 \mathrm{a}-4 \mathrm{~h}$ demonstrates the corresponding estimated spectra for $\eta$ and NMO velocity from shifted hyperbola approximation (equations 7 and 8), rational approximation (equations 10 and 11), three-parameter approximation (equations 15 and 18), and acceleration approximation (equations 20 and 21), respectively. The spectra are constructed in analogous way to Casasanta and Fomel (2011). Due to the numerical artifacts resulting from small local slopes at small offsets where the expressions become unstable, we filtered out the small-offset estimates. The yellow and red dots indicate the exact effective $\eta \mathrm{s}$ and the exact effective NMO velocities used for forward modeling, respectively.

To have a better comparison between the accuracy of estimated kinematic attributes from different approximations, we picked the maximum estimated attributes for the main time horizons in each spectrum and compared them with the exact values used in forward modeling. Figure 5a and 5b shows the comparison of effective $\eta \mathrm{s}$ and effective NMO velocities obtained by the application of different approximations using the proposed approach.

We observed that the application of all approximations results in underestimated $\eta \mathrm{s}$ and overestimated NMO velocities in the example. Rational approximation has the highest accuracy, whereas the acceleration approximation has the lowest accuracy. The error in effective $\eta$ s increases with time/depth, whereas the error in NMO velocity decreases with time/depth for all approximations. This observation is different from the observations of Stovas and Fomel (2016) stating that "the error in NMO velocity increases with depth for all approximations." The obtained results confirm the comparable accuracy of acceleration approximation with three-parameter approximation, as pointed out by Golikov and Stovas (2012).

\section{COMPARISON WITH MULTIPARAMETER SEMBLANCE}

In the previous section, we demonstrated the proposed approach on a synthetic data example and compared the accuracy of different approximations. In this section, we first estimated the kinematic attributes for the same synthetic data using traditional multiparameter semblance technique. Then, we compared the computational

Table 1. The NMO velocities and inhomogeneity parameters $(\eta)$ used in forward modeling to generate the VTI anisotropic CMP gather shown in Figure $2 b$.

\begin{tabular}{lcl}
\hline Interface & $V_{\mathrm{NMO}}(\mathrm{m} / \mathrm{s})$ & $\eta$ \\
\hline 1 & 1800 & 0.04 \\
2 & 1850 & 0.071 \\
3 & 1900 & 0.062 \\
4 & 1950 & 0.093 \\
5 & 2000 & 0.084 \\
6 & 2050 & 0.115 \\
7 & 2100 & 0.106 \\
8 & 2150 & 0.137 \\
9 & 2200 & 0.128 \\
10 & 2250 & 0.159 \\
11 & 2300 & 0.15 \\
\hline
\end{tabular}


time and the accuracy of the results obtained by semblance inversion and by the proposed approach.

For simultaneous search/inversion of the kinematic attributes using multiparameter semblance inversion, we defined 50 values for NMO velocities in the range of $1000-2500 \mathrm{~m} / \mathrm{s}$ and 50 values for $\eta \mathrm{s}$ in the range of $0.01-0.25$. Figure $6 \mathrm{a}-6 \mathrm{~d}$ shows four NMO velocity- $\eta$ spectra, indicating the inversion results for four different times
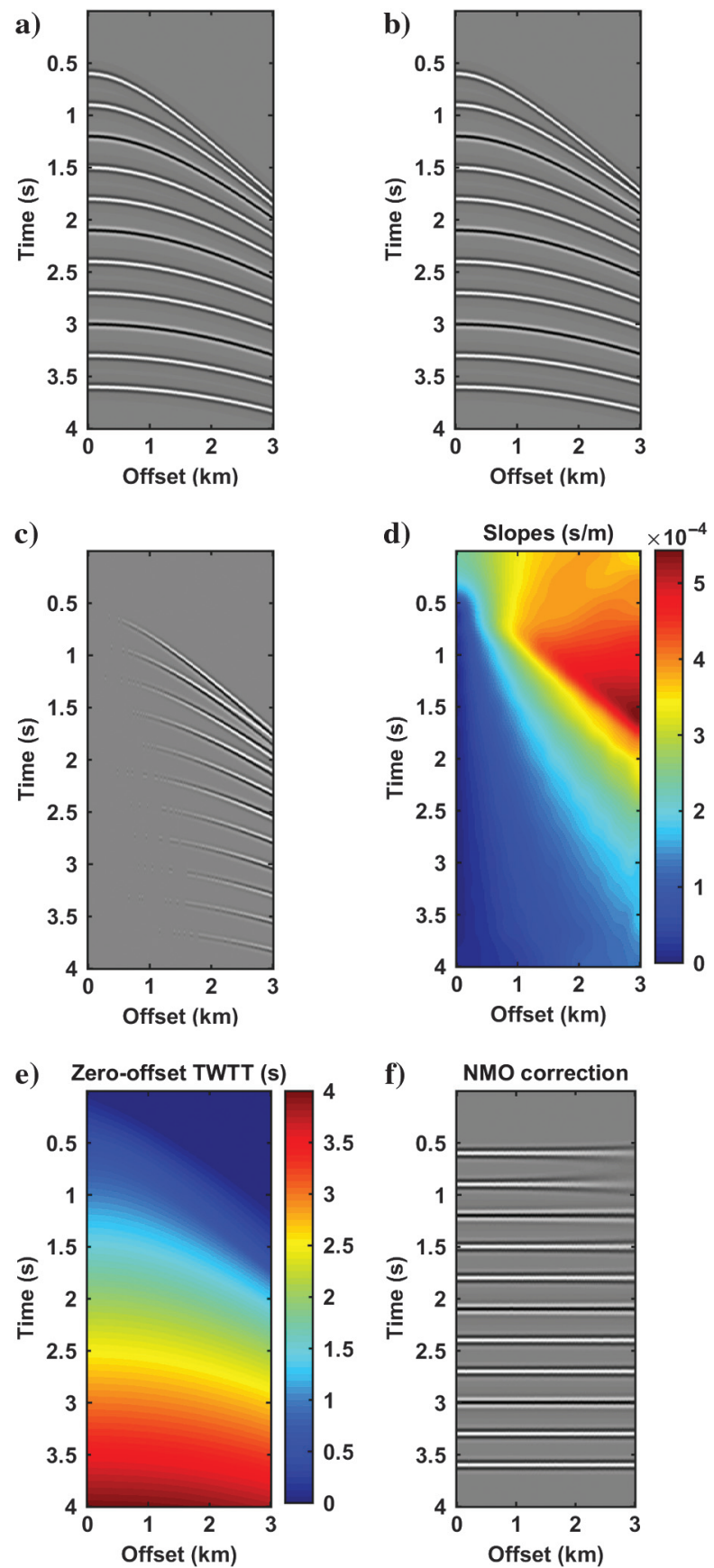

Figure 2. Generated (a) isotropic and (b) anisotropic CMP gather based on the kinematic attributes expressed in Table 1, (c) the amplitude difference between the isotropic and anisotropic CMP gathers, (d) local slopes estimated by plane-wave destructor, (e) zerooffset TWTTs by predictive painting, and (f) flattened data using the estimated zero-offset TWTT for the anisotropic CMP gather. using rational approximation. It is difficult to recognize the maximum semblance values by visual observation within the spectra. The maximum values were chosen by automatic picking. The asterisks in the spectrums show the picked values. One can observe that the width of NMO velocities in each spectra is noticeably narrower than the width of $\eta \mathrm{s}$. It confirms that the uncertainty in the inversion of NMO velocity is smaller than the uncertainty in the inversion of $\eta$.

Similar to the previous section, to quantify the accuracy of the estimated kinematic attributes from different approximations using multiparameter semblance inversion, we used the automatically picked values corresponding to the main time horizons in each spectrum and compared them with the exact values used for forward modeling. Figure $7 \mathrm{a}$ and $7 \mathrm{~b}$ shows the comparison of the effective $\eta \mathrm{s}$ and the effective NMO velocities obtained by the application multiparameter semblance inversion for the shifted hyperbola approximation (equations 4 and 8), rational approximation (equation 9), three-parameter approximation (equations 13 and 18), and acceleration approximation (equation 19).

Based on the obtained results shown in Figure 7, the rational approximation has the highest accuracy, whereas the acceleration approximation has the lowest. The deviations from the exact values used for forward modeling in the estimated effective $\eta \mathrm{s}$ are not stable with time/depth (there is no a specific trend), whereas the error in NMO velocities decreases with time/depth for all approximations. The estimations from multiparameter semblance inversion confirm the estimations from the proposed approach.

Accuracy comparison between the results from the proposed and multiparameter semblance inversion techniques (Figures 5 and 7) is difficult by visual inspection; therefore, we computed the relative errors in the estimation of kinematic attributes using both techniques for different approximations. Tables 2 and 3 show the computed errors in the estimation of effective $\eta \mathrm{s}$ and NMO velocities, respectively. It is shown that the overall relative error using the proposed inversion technique is less than the multiparameter semblance inversion technique for the presented example.

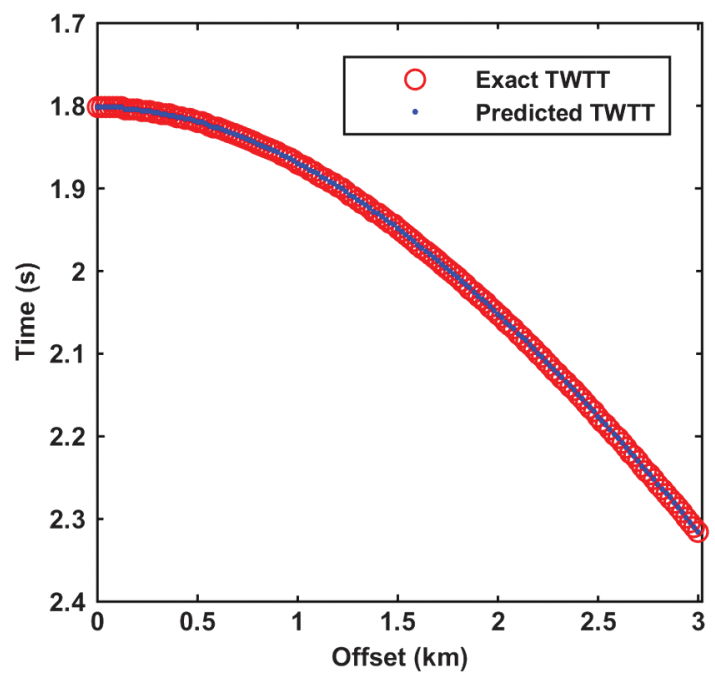

Figure 3. Comparison between the exact arrival times and the predicted arrival times for interface 5 of the model expressed in Table 1 and Figure $2 b$. 
The most important advantage of velocity-independent imaging/ inversion techniques over the traditional techniques is related to their time efficiency. Oriented techniques can be orders of magnitudes faster than the traditional techniques as confirmed in Table 4, which shows the comparison of the computational times by the application of the proposed technique and semblance inversion using the same desktop computer.

\section{COMPARISON WITH THE PREVIOUS ORIENTED TECHNIQUES}

In the previous sections, we suggested that the proposed technique is a further improvement and simplification of the previous oriented/velocity-independent inversion techniques for the estimation of kinematic attributes in VTI media. In this section, the same synthetic anisotropic CMP gather (Figure 2b) is used to quantify the accuracy in the inversion of the attributes using the previous techniques. The shifted hyperbola and rational approximations, which are considered in the presented and the previous approaches (Fomel, 2007a; Stovas and Fomel, 2016), are used for the demonstration.

\section{Shifted hyperbola approximation}

The second derivative of TWTT with respect to offset (curvature), which is the derivative of local slopes with respect to offset, is one of the inputs in the estimation of kinematic attributes using shifted hyperbola approximation in the previous velocity-independent inversion techniques (Fomel, 2007a; Stovas and Fomel, 2016). The curvature is estimated by the application of the chain rule (Bóna, 2011; Casasanta and Fomel, 2011)

$$
q=\frac{\partial p}{\partial x}+p \frac{\partial p}{\partial t}
$$

where $q$ denotes the second derivative of TWTT with respect to offset. The gradient components of local slopes in offset and time directions $(\partial p / \partial x, \partial p / \partial t)$ are estimated using numerical differentiation. The curvature estimated from equation 22 is shown in Figure $8 \mathrm{a}$. Another way to estimate the curvature is to use semblance analysis (see the "Discussion" section). The kinematic attributes, including zero-offset TWTT, derived by Fomel (2007a), and a)

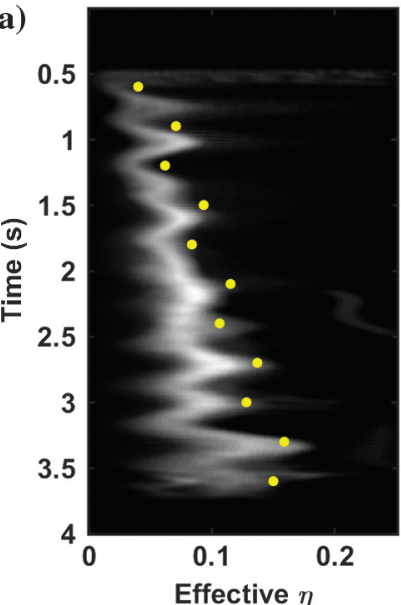

e)

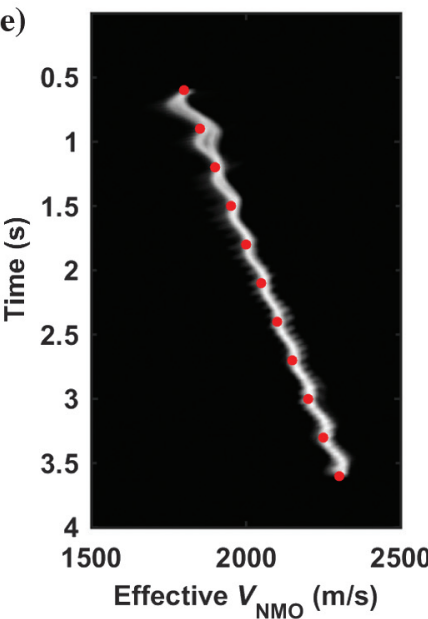

b)
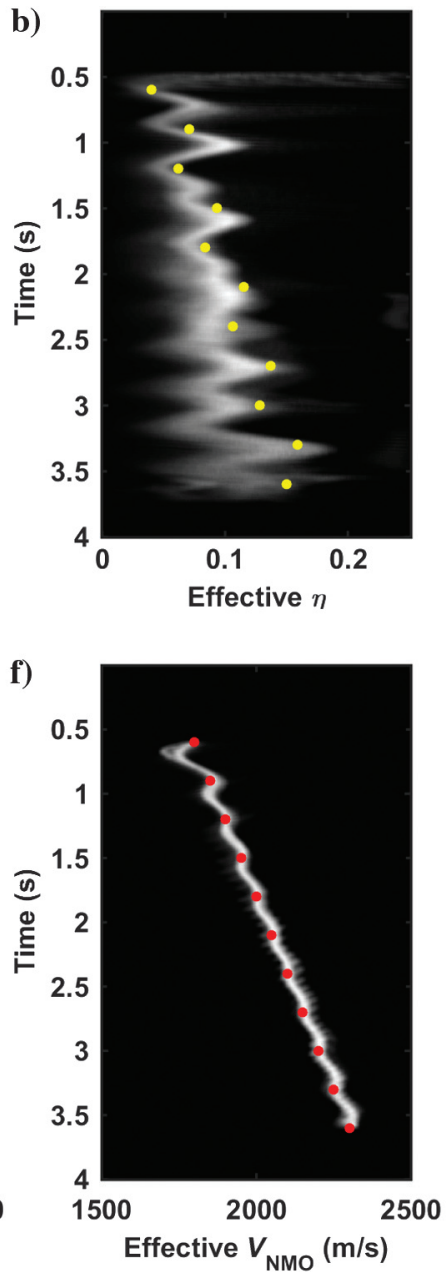

c)
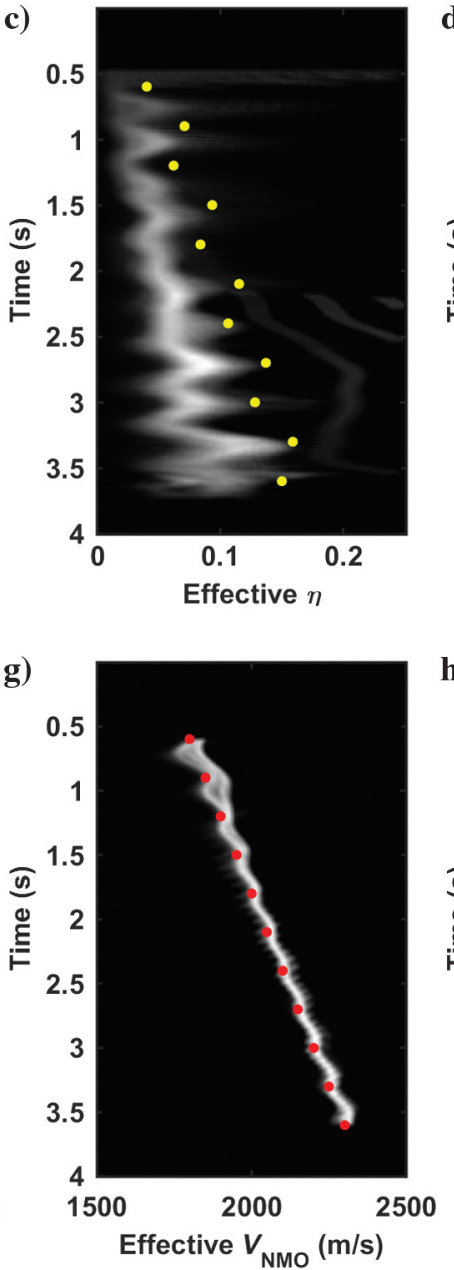

d)
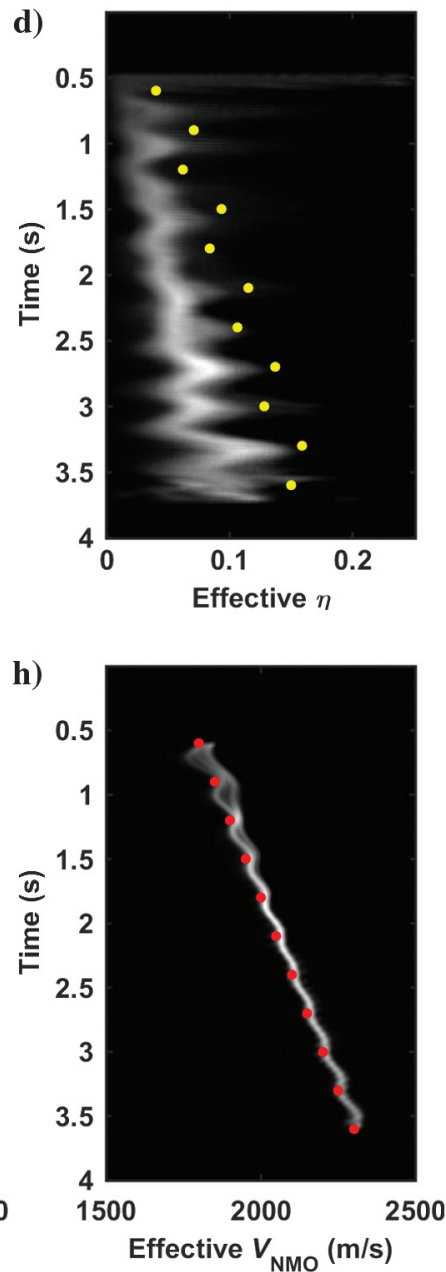

Figure 4. Estimated $\eta$ from (a) shifted hyperbola, (b) rational, (c) three-parameter and (d) acceleration approximations, and estimated NMO velocity from (e) shifted hyperbola, (f) rational, and (g) three-parameter and (h) acceleration approximations, by the application of the proposed technique for the anisotropic CMP gather shown in Figure 2b. Yellow and red dots indicate the exact effective $\eta$ s and NMO velocities used for forward modeling, respectively. 
Stovas and Fomel (2016) for shifted hyperbola approximation are expressed by

$$
\begin{gathered}
t_{0}=t-\frac{p x}{1+\sqrt{\frac{q x}{p}}}, \\
s=1+\frac{p(t-p x)-q x t}{\sqrt{q p^{3} x^{3}}}, \\
v^{2}=\frac{1}{t_{0}} \sqrt{\frac{q x^{3}}{p^{3}}} .
\end{gathered}
$$

In the above equations, $p, q, x, t, t_{0}, v$, and $s$ denote local slope, curvature, offset, TWTT, zero-offset TWTT, NMO velocity, and anisotropic parameter, respectively. The relation between $s$ and the nonhyperbolicity (anisotropy anellipticity) coefficient $\eta$ is given in equation 8 (Golikov and Stovas, 2012). Figure 8b shows the estimated zero-offset TWTT using equation 23. Figure $8 \mathrm{c}$ shows the result of flattening of the anisotropic gather shown in Figure $2 b$, using the estimated zero-offset TWTTs. It is observed that the data
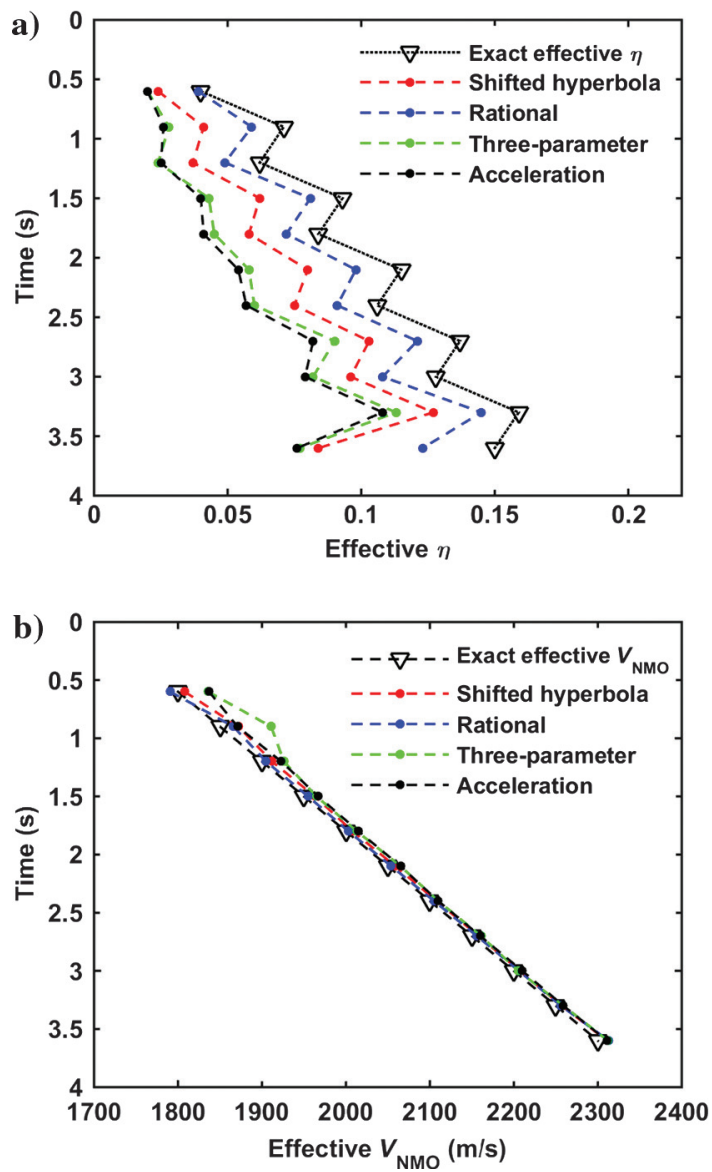

Figure 5. Comparison of (a) $\eta$ s and (b) NMO velocities for the main interfaces estimated from different approximations by the implementation of the proposed technique to the anisotropic CMP gather shown in Figure $2 b$. are not properly flatted in far-offsets, which are the area of interest for anisotropy studies.

Figure $9 \mathrm{a}$ and $9 \mathrm{~b}$ shows the estimated effective $\eta \mathrm{s}$ and the estimated NMO velocities for shifted hyperbola approximation from equations 24 and 25, respectively.

Comparison between the estimated attributes by the proposed technique (Figure $4 \mathrm{a}$ and $4 \mathrm{e}$ ) and by the previous techniques (Figure $9 \mathrm{a}$ and $9 \mathrm{~b}$ ) shows that accuracy in the inversion of the kinematic attributes from the proposed technique is noticeably higher than in the previous technique. This is due to the curvature independence of the proposed technique.

\section{Rational approximation}

The kinematic attributes ( $\eta$ and NMO velocity) derived by Stovas and Fomel (2016) for rational approximation are given in equations 9-12. In their approach, zero-offset TWTT $\left(t_{0}\right)$ is estimated by solving the following equation:

$$
t_{0}^{2}=\tau_{0}^{2}+t^{2}-p t x
$$

The $\tau_{0}^{2}$ must be estimated by solving the following fourth-degree equation (for $\tau_{0}^{2}$ ):

$$
a \tau_{0}^{8}+b \tau_{0}^{6}+c \tau_{0}^{4}+d \tau_{0}^{2}+e=0,
$$

where

$$
a=16 \text {, }
$$
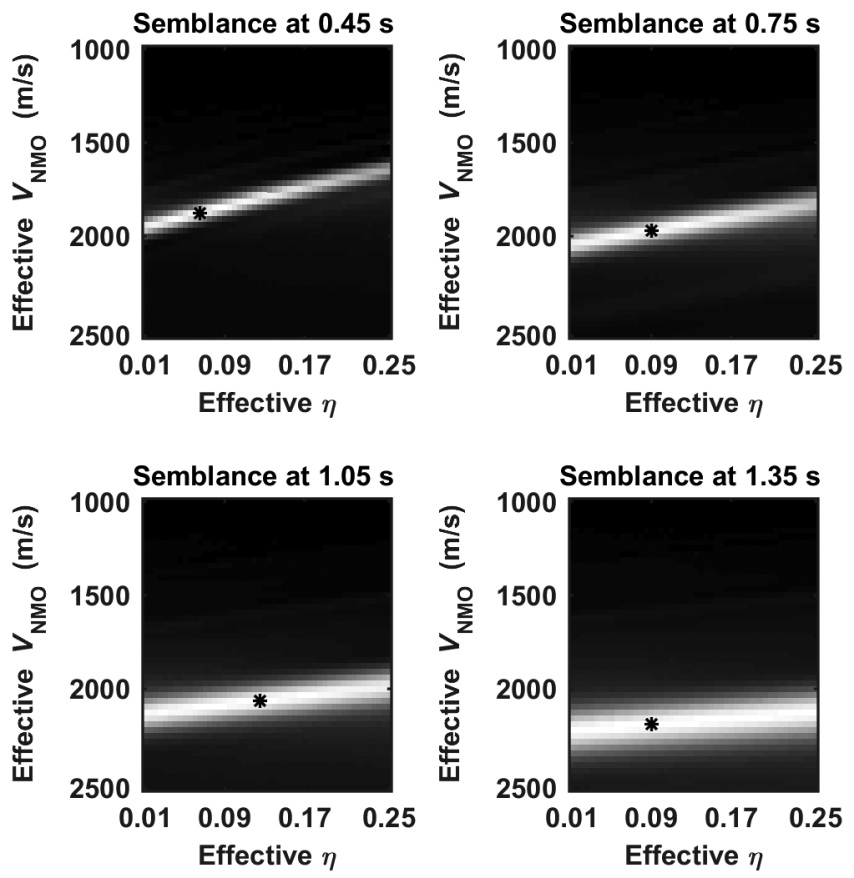

Figure 6. Inversion results of NMO velocity and effective $\eta$ for four different times by the application of multiparameter semblance inversion to the anisotropic CMP gather shown in Figure $2 b$ using rational approximation. Asterisks show the maximum values of semblance in each spectrum. 


$$
\begin{gathered}
b=-8 D x, \\
c=12 D p t x^{2}, \\
d=D t^{2} x^{2}[-D+4 p(t-p x)], \\
D=p t-p^{2} x-q t x .
\end{gathered}
$$

Solution of equation 27 is provided in Appendix A. Figure 10a shows the estimated zero-offset TWTT from equation 26. Figure $10 \mathrm{~b}$ shows the result of flattening of the anisotropic CMP gather shown in Figure 2b, using the estimated zero-offset TWTTs. It is observed that the data are not properly flattened in near and far offsets.

Figure $11 \mathrm{a}$ and $11 \mathrm{~b}$ shows the estimated effective $\eta \mathrm{s}$ and the estimated NMO velocities for rational approximation from equations 10 and 11 (Stovas and Fomel, 2016), respectively. Accuracy in the inversion of the kinematic attributes using the proposed technique is noticeably higher than the previous technique. This higher resolution is due to: (1) the curvature independence of the proposed approach, and (2) unique estimated values for zero-offset TWTT $\left(t_{0}\right)$, rather than nonunique answers obtained by the solution of
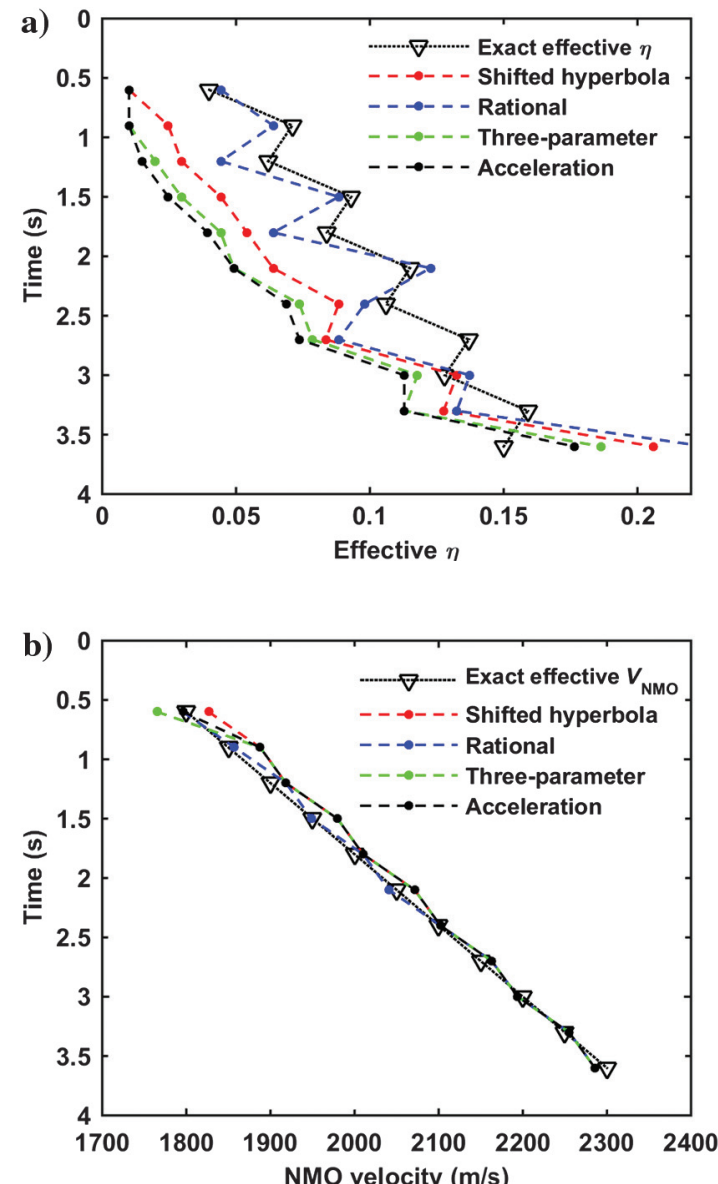

Figure 7. Comparison of (a) effective $\eta$ s and (b) NMO velocities estimated from different approximations, by the application of multiparameter semblance to the anisotropic CMP gather shown in Figure $2 b$. the fourth-degree polynomial for $t_{0}^{2}$ (or $\tau_{0}^{2}$ ) proposed in the previous technique (see Appendix A).

\section{EXAMPLE BASED ON REAL DATA}

Because we did not have access to anisotropic data, we modified a marine CMP gather recorded in a largely isotropic region by Geoscience Australia in 1996 over the North West Shelf, Western Australia, shown in Figure 12a. The anisotropic effects are injected into the original gather to examine the performance of the proposed algorithm in real data situation. The following steps describe the modification:

1) The NMO velocities of the isotropic CMP gather (Figure 12a) were manually picked.

2) The NMO correction was applied to the data by the application of predictive painting (Figure 12b).

3) A range of $\eta$ s between 0 and 0.2 was defined.

4) Given the NMO velocities and the defined values for $\eta$, anisotropic modeling (Bóna et al., 2008) was done to design an inverse NMO operator (Figure 12c).

5) The anisotropic CMP gather was made by the application of the inverse NMO operator (Figure 12d).

Table 2. Relative error $(\%)$ in the estimation of effective $\eta$ for the CMP gather shown in Figure $2 \mathrm{~b}$ using the proposed and multiparameter semblance inversion techniques.

Semblance inversion Proposed inversion

\begin{tabular}{lll} 
Shifted hyperbola & 40 & 32 \\
Rational & 18 & 13 \\
Three parameter & 51 & 46 \\
Acceleration & 49 & 49 \\
\hline
\end{tabular}

Table 3. Relative error (\%) in the estimation of NMO velocity for the CMP gather shown in Figure $2 b$ using the proposed and multiparameter semblance inversion techniques.

Semblance inversion Proposed inversion

\begin{tabular}{lll}
\hline Shifted hyperbola & 0.85 & 0.51 \\
Rational & 0.4 & 0.35 \\
Three parameter & 0.75 & 0.82 \\
Acceleration & 0.9 & 1 \\
\hline
\end{tabular}

Table 4. Comparison of computational time between the proposed technique and multiparameter semblance inversion, for the whole CMP gather shown in Figure $2 \mathrm{~b}$.

Proposed technique Semblance technique

Computational time $15 \mathrm{~s} \quad 2 \mathrm{~h}$ 


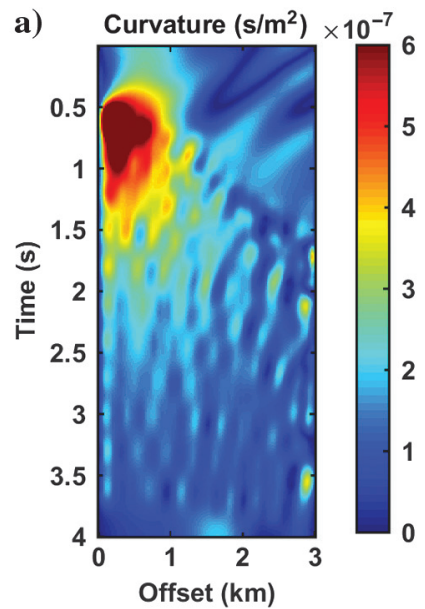

b) Zero-offset TWTT (s)
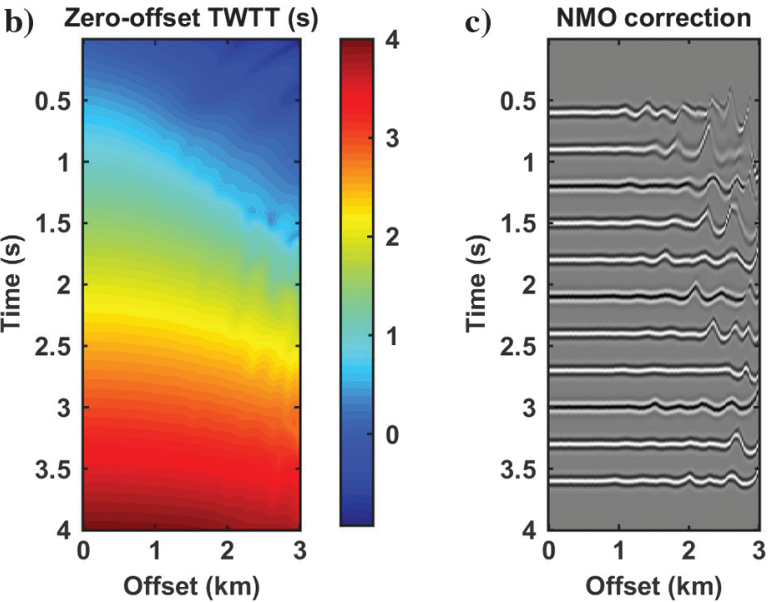

Figure 8. (a) Curvatures estimated from equation 22, (b) zero-offset TWTTs estimated from equation 23 (Fomel, 2007a; Stovas and Fomel, 2016), and (c) the NMO-corrected CMP gather using the estimated zero-offset TWTTs.
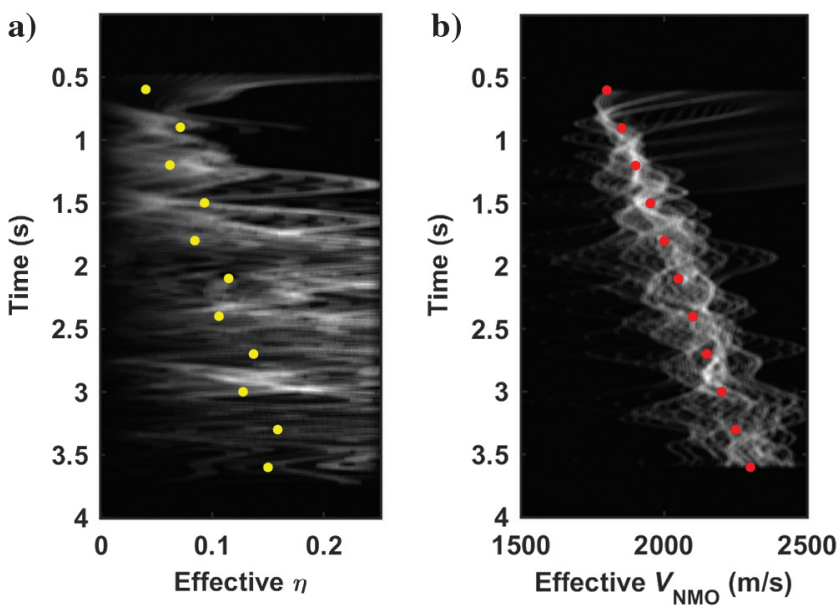

Figure 9. Estimated (a) effective $\eta$ s and (b) NMO velocities for shifted hyperbola approximation by the application of equations 24 and 25 , respectively. The indicated dots show the exact values of the kinematic attributes used for forward modeling to generate the anisotropic CMP gather shown in Figure $2 b$.
The modified CMP gather is suitable to examine the performance of the proposed inversion technique for a realistic survey. Another advantage of the use of the CMP gather is the primary knowledge of the NMO velocities and the effective $\eta \mathrm{s}$; it helps to judge and quantify the final results. Here, we estimated the kinematic attributes by implementing the proposed technique and multiparameter semblance inversion for rational approximation that had the highest accuracy among the other approximations presented in this paper. Then, we compared the accuracy of the obtained results by the application of both techniques.

Figure 13a and 13b shows the corresponding local slopes and zerooffset TWTTs for the CMP gather shown in Figure 12d, estimated using plane-wave destructor and predictive painting, respectively.

Figure $14 \mathrm{a}$ and $14 \mathrm{~b}$ shows the corresponding results in the inversion of NMO velocities and the effective $\eta \mathrm{s}$, respectively. Green lines indicate the exact values of the attributes, red lines indicate the highest probable values estimated by the application of the pro-
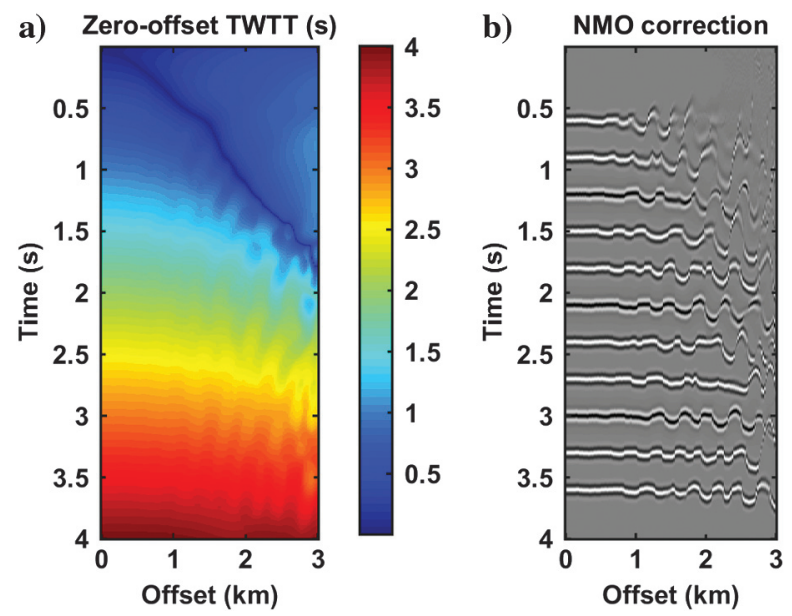

Figure 10. (a) Zero-offset TWTTs estimated from equation 26 (Stovas and Fomel, 2016) and (b) the corresponding NMO-corrected CMP gather using the estimated zero-offset TWTTs for the anisotropic CMP gather shown in Figure $2 b$.
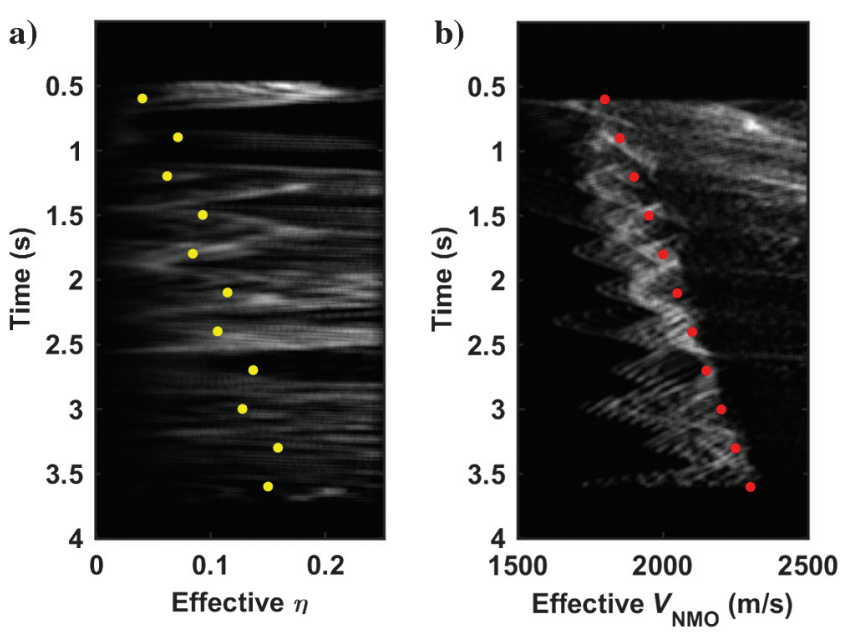

Figure 11. Estimated (a) effective $\eta$ and (b) NMO velocity for rational approximation by the application of equations 10 and 11 , respectively. The indicated dots show the exact values of the kinematic attributes used for forward modeling. 
posed technique, and yellow lines show the estimated values from multiparameter semblance inversion.

Table 5 shows the computed relative errors in the estimation of NMO velocities and effective $\eta \mathrm{s}$, respectively. Based on the achieved results, the overall relative error using the proposed inversion technique is smaller than the error using multiparameter semblance inversion technique. Computational time using the proposed technique was approximately $10 \mathrm{~s}$, whereas it was approximately $2 \mathrm{~h}$ using the semblance-inversion technique.

\section{SENSITIVITY TO NOISE}

Accuracy of any local slope based velocity-independent imaging/ inversion technique, depends on the precision of the estimated local slopes. We used plane-wave destructor to estimate the local slopes. In our experience, the plane-wave destructor is not as sensitive as other local slope estimation techniques that use numerical differentiation (Schleicher et al., 2009) to white noise. It is because planewave destructor relies on (1) smoothing the seismic data and (2) use of regularization (Fomel, 2002, 2007b); however, if the level of
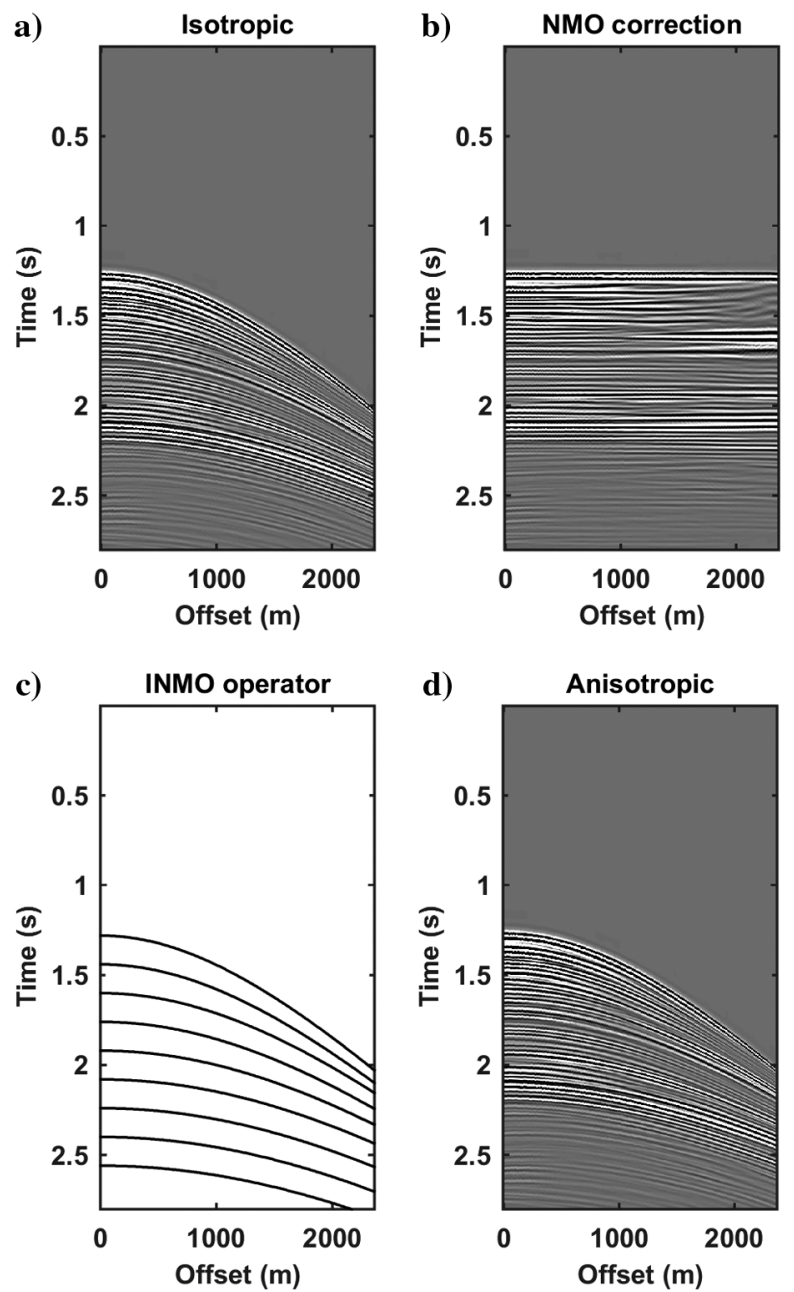

Figure 12. (a) Original CMP gather from North West Shelf, Western Australia, (b) velocity-independent NMO-corrected CMP gather by the application of predictive painting, (c) anisotropic inverse NMO operator, and (d) inverse NMO-corrected (anisotropic) CMP gather by the application of the anisotropic operator. noise is high, the accuracy of the local slopes estimated by planewave destructor will also be affected.

To examine the performance of the technique in the presence of noise, we contaminated the synthetic CMP gather used in the first example with band limited white noise with the frequency range of $5-150 \mathrm{~Hz}$, so that the signal-to-noise ratio $(\mathrm{S} / \mathrm{N})$ is 10 . This level of $\mathrm{S} / \mathrm{N}$ is fairly acceptable for the residual noise left in a gather/section after pre-processing and denoising. We used the following equation to scale the $\mathrm{S} / \mathrm{N}$ :

$$
\frac{S}{N}=\frac{E_{S}}{E_{N}}=\frac{\sum_{i=1}^{n} \sum_{j=-m}^{m} A_{S_{i, j}}^{2}}{\sum_{i=1}^{n} \sum_{j=-m}^{m} A_{N_{i, j}}^{2}},
$$

where $A_{S}$ and $A_{N}$ denote the amplitude of signal and noise in each sample, respectively. Indices $i$ and $j$ denote trace and sample num-
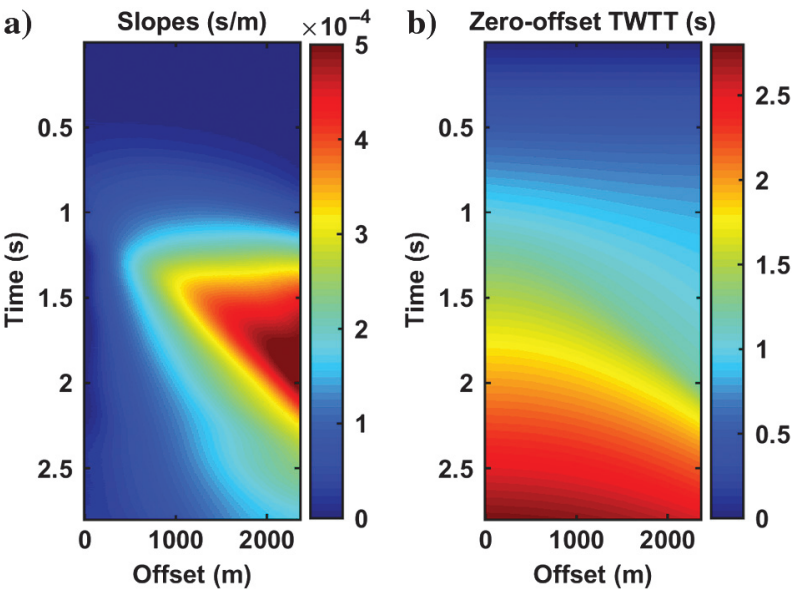

Figure 13. (a) Local slopes estimated by plane-wave destructor and (b) zero-offset TWTTs estimated by predictive painting, for the marine CMP gather shown in Figure 12d.
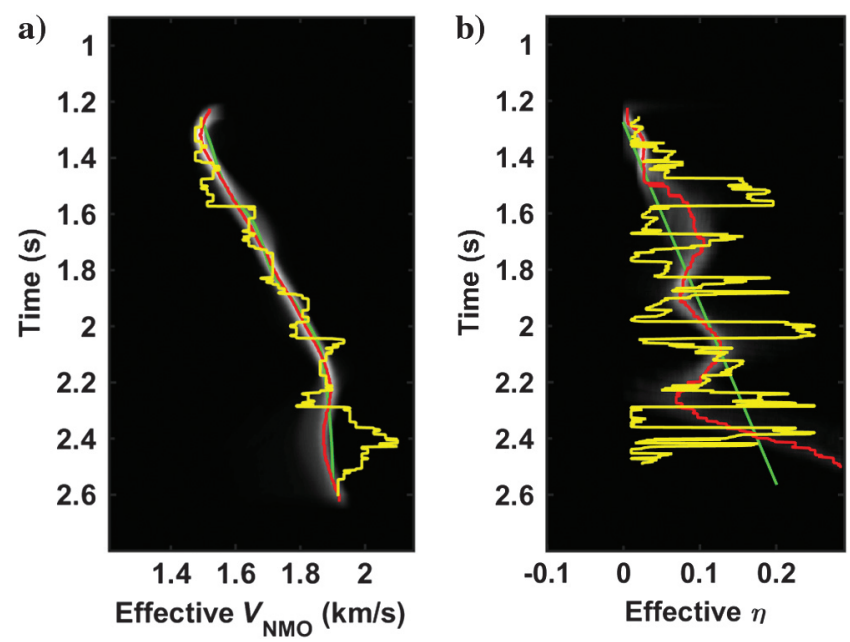

Figure 14. (a) Effective $\eta$ s and (b) NMO velocities estimated using rational approximation for the CMP gather shown in Figure 12d. Green lines indicate the exact values, red lines indicate the estimated values by the application of the proposed technique, and yellow lines indicate the values estimated by the application of routine multiparameter semblance analysis. 
ber in each trace within the window of each seismic event $(2 m)$, respectively. As the purpose of this example is to study the sensitivity of the proposed technique to noise, we only used the rational approximation that had the highest accuracy compared with the other approximations presented in this paper. Figure $15 \mathrm{a}-15 \mathrm{c}$ shows the noisy synthetic data, the corresponding estimated local slopes and zero-offset TWTTs using plane-wave destructor and predictive painting, respectively. Figure $15 \mathrm{~d}$ shows the NMO-corrected data using the estimated zero-offset TWTTs. Note that to have higher

Table 5. Relative error $(\%)$ in the estimation of NMO velocity and effective $\boldsymbol{\eta}$ for the field CMP gather shown in Figure 12d from the multiparameter semblance and the proposed techniques.

\begin{tabular}{lcc}
\hline & Semblance inversion & Proposed inversion \\
\hline NMO velocity & 4.5 & 0.006 \\
Effective $\eta$ & 111 & 57 \\
\hline
\end{tabular}
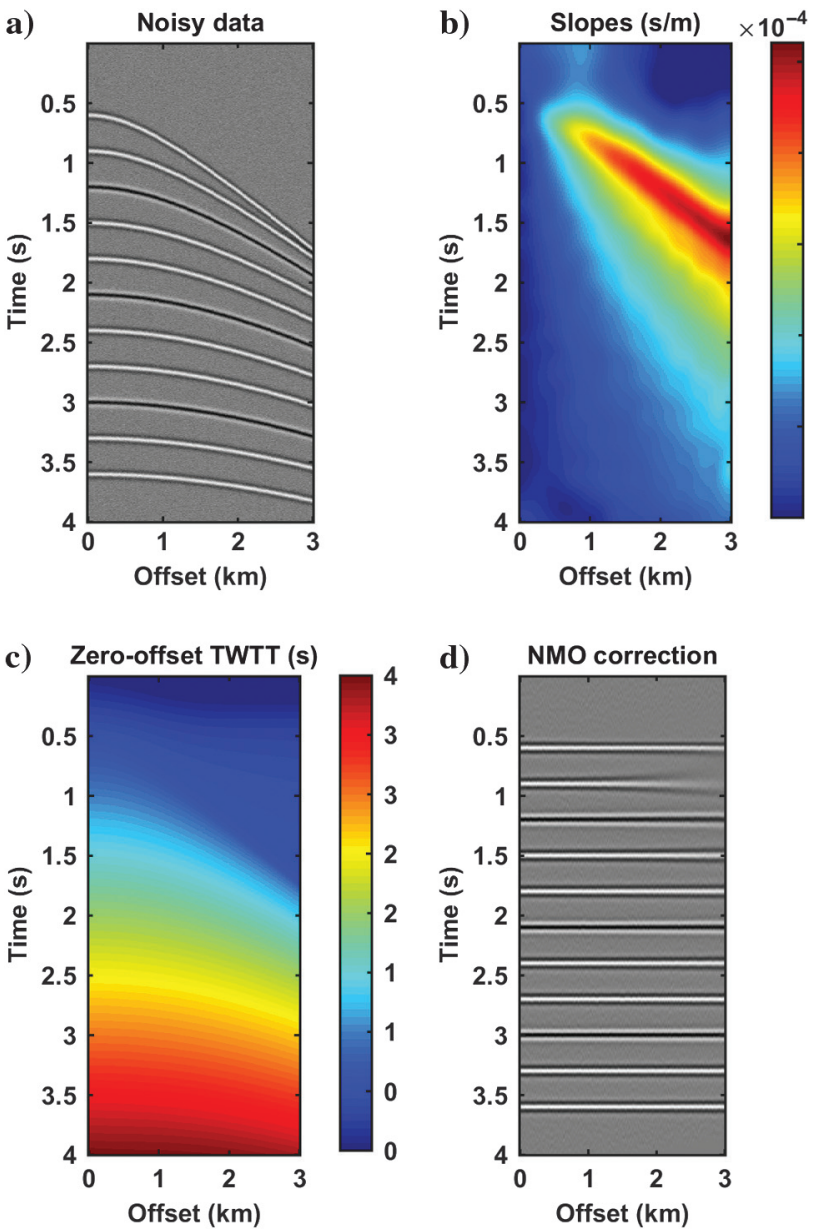

Figure 15. (a) Noisy CMP gather generated based on the kinematic attributes in Table 1 and contaminated with band limited white noise $(\mathrm{S} / \mathrm{N}=10)$, (b) local slopes estimated by plane-wave destructor, (c) zero-offset TWTTs by predictive painting, and (d) the corresponding velocity-independent NMO-corrected CMP gather. accuracy in the estimation of local slopes in the presence of noise, we increased the differentiation order from 6 (used in all previous examples) to 14. Local slopes estimation took 1 min using order 14

Figure 16a and 16b shows the estimated NMO velocities and effective $\eta \mathrm{s}$ for the noisy CMP gather shown in Figure 15a from the proposed technique, respectively.

Comparison between the results from the noise-free and noisy CMP gathers shows that the proposed technique is not noticeably affected by the use of local slopes estimated with higher order differentiation (order 14). To quantify this, we computed the relative error in the estimation of kinematic attributes using the presented technique for the noise-free and the noisy data. The comparison is expressed in Table 6.

\section{DISCUSSION}

In the presented research, we showed that removing the second derivative of TWTT with respect to offset (curvature) leads to more accurate estimation of kinematic attributes in VTI media. The reason for the higher accuracy in the estimation of the attributes is due to the reduction of numerical errors caused by the calculation of curvature.

Estimation of curvature using numerical differentiation (equation 22) is unstable because finite differences made by the implementation of numerical differentiation act as high-pass filters, which increase the high-frequency noise, especially in the presence of
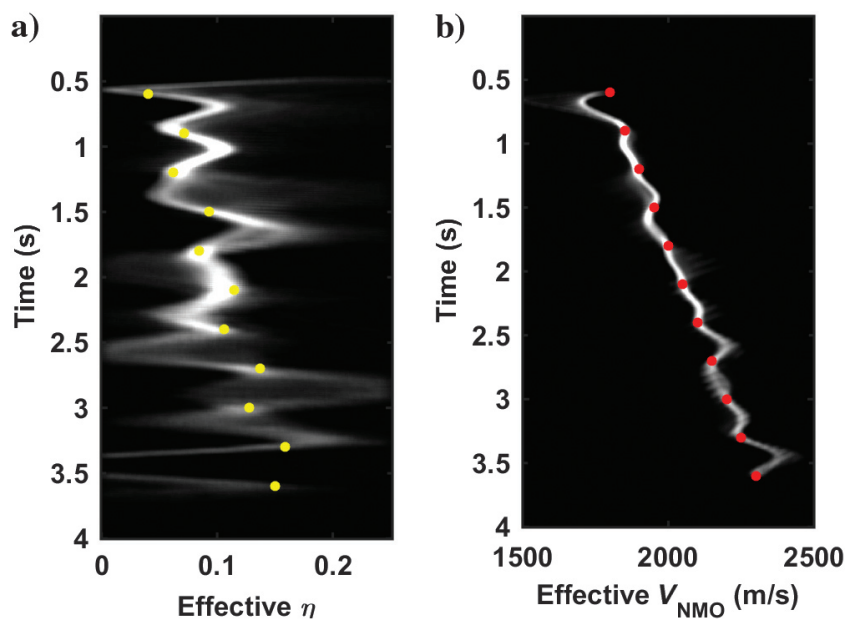

Figure 16. Estimated (a) effective $\eta \mathrm{s}$ and (b) NMO velocities by the application of the proposed technique using rational approximation to the noisy CMP gather shown in Figure 15a. The indicated dots show the exact values of the kinematic attributes used for forward modeling.

Table 6. Relative error $(\%)$ in the estimation of NMO velocity and effective $\boldsymbol{\eta}$ for the noise-free and noisy CMP gathers shown in Figures $2 b$ and 15a.

Noise-free data

Noisy data

\begin{tabular}{lcr}
\hline NMO velocity & 0.35 & 0.44 \\
Effective $\eta$ & 13 & 12.08 \\
\hline
\end{tabular}


noise (Casasanta and Fomel, 2011). There are usually enough samples in time direction to negate this high-pass filter; however, in the offset direction, it is not the case, specifically in far offsets that are more important to study anisotropy/nonhyperbolicity, where the seismic events can approach a line (curvature disappears). Taking spatial aliasing into account, curvature estimation can be even a more challenging task. To avoid the effect of spatial aliasing, interpolation can be applied to the data prior to curvature estimation.

The study of different techniques for curvature estimation, specifically in the presence of noise, is still an open task (Bóna, 2011). Semblance analysis using the Taylor expansion is another possibility for the curvature estimation. To do so, TWTT must be expanded around each point of a seismic gather/section. A scanning window with a defined number of traces should be defined and used. The corresponding expansion is given by

$$
t(h \pm \Delta x)=t(h)+p \Delta x+\frac{1}{2} q \Delta x^{2}+\ldots .
$$

Here, $t(h)$ is the TWTT in a given offset $(h)$ in a seismic gather/record that is located at the center of the scanning window, $t(h \pm \Delta x)$ is the expanded TWTT with a certain distance $(\Delta x)$ from the central trace in the scanning window, $p$ is the estimated slopes by planewave destructor or by another slope estimation technique, and $q$ denotes the desired curvature. Analogous to the procedure previously explained for multiparameter semblance analysis, a range of possible curvatures should be defined for scanning. There are the following limitations in curvature estimation using semblance analysis and Taylor expansion:

1) computational time that is very high/expensive

2) sensitivity to the number of traces chosen in the scanning window

3) instability in the estimation of curvature in far offsets, where curvature can be almost disappeared from the seismic events.

Considering these limitations and difficulties in the estimation of curvature with high accuracy, the presented research provides an improved approach by replacing the curvature estimates from the previous works with the corresponding zero-offset TWTT via predictive painting.

Ideally, the algorithm's input must be a pre-processed denoised data; however, it is potentially still possible to perform the proposed algorithm to a data containing higher levels of band limited random noise. To do so, one can update the estimated slopes, by the use of predictive painting and estimation of traveltime curves using time warping, as explained in the previous sections. This process can be repeated, and in principle the higher the number of iterations, the higher the accuracy of the updated local slopes

We also note that, while zero-offset TWTTs are the inputs of the proposed approach, which were computed by predictive painting in this paper, one can use another horizon picking technique (Lomask et al., 2006; Wu and Hale, 2014) for providing the zero-offset TWTTs.

\section{CONCLUSIONS}

We proposed a velocity-independent algorithm for the inversion of kinematic attributes in VTI media. The algorithm is the further improved and simplified version of the previous velocity-independent algorithms. The algorithm does not require a prior knowledge of a velocity model. The only initial requirements of the technique are local slopes in each point and the corresponding zero-offset TWTTs. The main advantages of the algorithm are its curvature independence and time-efficiency. Curvature dependence of the previous techniques was replaced by the use of predictive painting technique to estimate the zero-offset TWTTs. Higher accuracy in the inversion of the attributes by the proposed velocity-independent approach over the previous velocity-independent approaches was quantified using a synthetic data example. It was also demonstrated that the presented method can be orders of magnitude faster than routine multiparameter semblance inversion for the estimation of kinematic attributes in VTI media.

Comparison between the inverted kinematic attributes obtained by the application of the proposed technique to a numerical transversely isotropic model from shifted-hyperbola approximation, rational approximation, three-parameter approximation, and acceleration approximation shows that the rational approximation has the highest accuracy, whereas the acceleration approximation has the lowest accuracy for $\eta$ s and NMO velocities. Regardless of approximation type, it is demonstrated that the accuracy in the inversion of NMO velocities is higher than the accuracy in the inversion of $\eta \mathrm{s}$. As opposed to some previous observations, the results show that error in NMO velocity inversion does not necessarily increase with depth.

The proposed technique can be used as an alternative for the inversion of the kinematic attributes in VTI media, specifically for offshore data that do not contain considerable level of noise. Further development of the technique involves extension of the approach to other approximations and to simplify the corresponding formulations for generalized moveout approximation and also to estimate the interval parameters (in time and depth) from the effective estimated parameters.

\section{ACKNOWLEDGMENTS}

The work has been supported by the Deep Exploration Technologies Cooperative Research Centre (DET CRC), whose activities are funded by the Australian Government's Cooperative Research Centre Program. This is DET CRC document 2015/760. Authors would like to acknowledge Geoscience Australia for providing the marine data. We would like to acknowledge Madagascar Open-Source Project for providing the multidimensional data analysis package. The authors would also like to thank S. Fomel, A. Stovas, and K. Chambers for their comments and suggestions. A special acknowledgement goes to the editorial committee, M. Sacchi, V. Socco, and I. Jones, and four anonymous reviewers, whose suggestions and comments have improved the clarity of the paper.

\section{APPENDIX A}

\section{SOLUTION OF ZERO-OFFSET TWTT FOR RATIONAL APPROXIMATION}

Four possible solutions for $\tau_{0}^{2}$, expressed in equation 27 in the main text, are given by

$$
\tau_{01,2}^{2}=\frac{b}{4 a}-z \pm \frac{1}{2} \sqrt{-4 z^{2}-2 m-\frac{n}{z}}
$$




$$
\tau_{03,4}^{2}=\frac{b}{4 a}-z \pm \frac{1}{2} \sqrt{-4 z^{2}-2 m+\frac{n}{z}} .
$$

The parameter $z$ is given by

$$
z=\frac{1}{2} \sqrt{-\frac{2}{3} m+\frac{1}{3 a}\left(l+\frac{w}{l}\right)},
$$

where

$$
l=\sqrt[3]{\frac{y+\sqrt{y^{2}-4 w^{3}}}{2}} .
$$

Parameters $w$ and $y$ are defined by

$$
\begin{gathered}
w=c^{2}-3 b d+12 a e, \\
y=2 c^{3}-9 b c d+27 b^{2} e+27 a d^{2}-72 a c e,
\end{gathered}
$$

where

$$
\begin{gathered}
m=\frac{8 a c-3 b^{2}}{8 a^{2}}, \\
n=\frac{b^{3}-4 a b c+8 a^{2} d}{8 a^{3}} .
\end{gathered}
$$

\section{REFERENCES}

Alkhalifah, T., and I. Tsvankin, 1995, Velocity analysis for transversely isotropic media: Geophysics, 60, 1550-1566, doi: 10.1190/1.1443888.

Asgharzadeh, M., D. Nadri, and A. Bóna, 2014, Inversion based accuracy comparison of nonhyperbolic moveout approximations for P-waves in VTI media: 76th Annual International Conference and Exhibition, EAGE, Extended Abstracts, doi: 10.3997/2214-4609.20140989.

Blias, E., 2007, Long-spread length approximations to NMO function for a multi-layered subsurface: Recorder, 3, 36-42.

Blias, E., 2009, Long-offset NMO approximations for a layered VTI model. Model study: 79th Annual International Meeting, SEG, Expanded Abstracts, 3745-3749, doi: 10.1190/1.3255647.

Bóna, A., I. Bucataru, and A. Slawinski, 2008, Inversion of ray velocity and polarization for elasticity tensor: Journal of applied Geophysics, $\mathbf{6 5}, 1-5$, doi: 10.1016/j.jappgeo.2008.01.004.

Bóna, A., 2011, Shot-gather time migration of planar reflectors without velocity model: Geophysics, 76, no. 2, S93-S101, doi: 10.1190/1.3549641.

Burnett, W., and S. Fomel, 2009a, 3D velocity-independent elliptically anisotropic moveout correction: Geophysics, 74, no. 5, WB129-WB136, doi: $10.1190 / 1.3184804$
Burnett, W., and S. Fomel, 2009b, Moveout analysis by time-warping: 79th Annual International Meeting, SEG, Expanded Abstracts, 3710-3714, doi: 10.1190/1.3255639.

Casasanta, L., and S. Fomel, 2011, Velocity-independent $\tau$ - $p$ moveout in a horizontally layered VTI medium: Geophysics, 76, no. 4, U45-U57, doi: 10.1190/1.3595776.

Castle, R. J., 1994, Theory of normal moveout: Geophysics, 59, 983-999, doi: $10.1190 / 1.1443658$

Cooke, D., A. Bóna, and B. Hansen, 2009, Simultaneous time imaging, velocity estimation and multiple suppression using local event slopes: Geophysics, 74, no. 6, WCA65-WCA73, doi: 10.1190/1.3242751.

De Bazelaire, E., 1988, Normal moveout revisited: Inhomogeneous media and curved interfaces: Geophysics, 53, 143-157, doi: 10.1190/1 .1442449 .

Fomel, S., 2002, Applications of plane-wave destruction filters: Geophysics, 67, 1946-1960, doi: 10.1190/1.1527095.

Fomel, S., 2007a, Velocity-independent time-domain seismic imaging using local event slopes: Geophysics, 72, no. 3, S139-S147, doi: 10.1190/1 .2714047 .

Fomel, S., 2007b, Shaping regularization in geophysical-estimation problems: Geophysics, 72, no. 2, R29-R36, doi: 10.1190/1.2433716.

Fomel, S., 2010, Predictive painting of 3D seismic volumes: Geophysics, 75, no. 4, A25-A30, doi: 10.1190/1.3453847.

Fomel, S., P. Sava, I. Vlad, Y. Liu, and V. Bashkardin, 2013, Madagascar: Open-source software project for multidimensional data analysis and reproducible computational experiments: Journal of Open Research Software, 1, e8, doi: 10.5334/jors.ag.

Fomel, S., and A. Stovas, 2010, Generalized nonhyperbolic moveout approximation: Geophysics, 75, no. 2, U9-U18, doi: 10.1190/1.3334323.

Fowler, P. J., A. Jackson, J. Gaffney, and D. Boreham, 2008, Direct nonlinear traveltime inversion in layered VTI media: 78th Annual International Meeting, SEG, Expanded Abstracts, 3028-3032.

Golikov, P., and A. Stovas, 2012, Accuracy comparison of nonhyperbolic moveout approximations for qP-waves in VTI media: Journal of Geophysics and Engineering, 9, 428-432, doi: 10.1088/1742-2132/9/4/428.

Lomask, J., A. Guitton, S. Fomel, J. Claerbout, and A. A. Valenciano, 2006 Flattening without picking: Geophysics, 71, no. 4, P13-P20, doi: 10 $.1190 / 1.2210848$.

Malovichko, A. A., 1978, A new representation of the traveltime curve of reflected waves in horizontally layered media: Applied Geophysics (in Russian), 91, 47-53.

Ottolini, R., 1983, Velocity independent seismic imaging: Stanford University, Technical report, Stanford Exploration Project, SEP-37.

Riabinkin, L. A., 1957, Fundamentals of resolving power of controlled directional reception (CDR) of seismic waves, in L. Lu, ed., Slant-stack processing: SEG, 36-60.

Rieber, F. 1936, A new reflection system with controlled directional sensitivity: Geophysics, 1, 97-106, doi: 10.1190/1.1437082.

Schleicher, J., J. C. Costa, L. T. Santos, A. Novais, and M. Tygel, 2009, On the estimation of local slopes: Geophysics, 74, no. 4, P25-P33, doi: 10 $.1190 / 1.3119563$.

Stovas, A., and S. Fomel, 2016, Mapping of moveout attributes using loca slopes: Geophysical prospecting, 64, 31-37, doi: 10.1111/1365-2478 .12284 .

Taner, M. T., S. Treitel, and M. Al-Chalabi, 2005, A new travel time estimation method for horizontal strata: 75th Annual International Meeting, SEG, Expanded Abstracts, 2273-2276, doi: 10.1190/1.2148170.

Taner, M. T., S. Treitel, M. Al-Chalabi, and S. Fomel, 2007, An offset dependent NMO velocity model: 69th Annual International Conference and Exhibition, EAGE, Extended Abstracts, P036, doi: 10.3997/2214-4609 .201401751

Wu, X., and D. Hale, 2014, Horizon volumes with interpreted constraints: Center for Wave Phenomena, Report CWP-812.

Yilmaz, O., 2001, Seismic data analysis, processing, inversion, and interpretation of seismic data: SEG. 